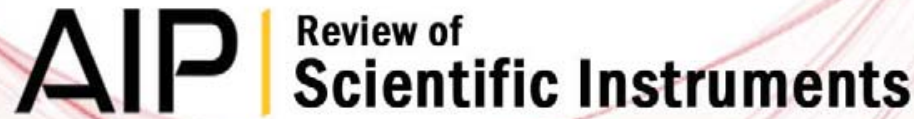

The fixed-bias Langmuir probe on the Communication/Navigation Outage Forecast System satellite: Calibration and validation

J. Klenzing and D. Rowland

Citation: Rev. Sci. Instrum. 83, 114501 (2012); doi: 10.1063/1.4766333

View online: http://dx.doi.org/10.1063/1.4766333

View Table of Contents: http://rsi.aip.org/resource/1/RSINAK/v83/i11

Published by the American Institute of Physics.

Additional information on Rev. Sci. Instrum.

Journal Homepage: http://rsi.aip.org

Journal Information: http://rsi.aip.org/about/about_the_journal

Top downloads: http://rsi.aip.org/features/most_downloaded

Information for Authors: http://rsi.aip.org/authors

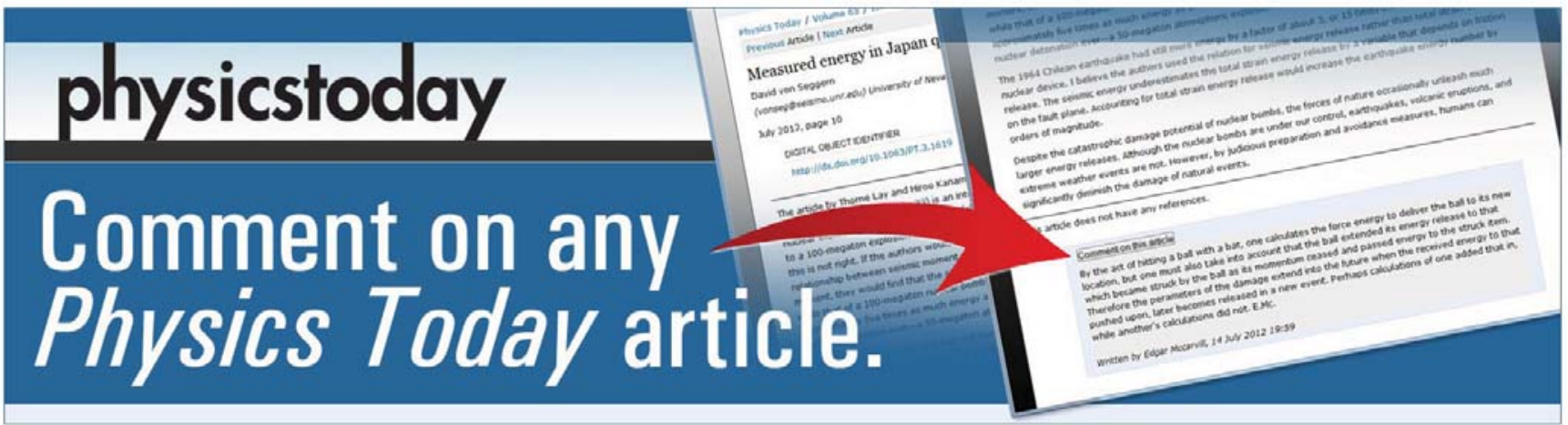




\title{
The fixed-bias Langmuir probe on the Communication/Navigation Outage Forecast System satellite: Calibration and validation
}

\author{
J. Klenzing ${ }^{\text {a) }}$ and D. Rowland \\ Space Weather Laboratory/Code 674, NASA Goddard Space Flight Center, Greenbelt, Maryland, USA
}

(Received 14 August 2012; accepted 22 October 2012; published online 12 November 2012)

\begin{abstract}
A fixed-bias spherical Langmuir probe is included as part of the Vector Electric Field Instrument (VEFI) suite on the Communication/Navigation Outage Forecast System (C/NOFS) satellite. C/NOFS gathers data in the equatorial ionosphere between 400 and $860 \mathrm{~km}$, where the primary constituent ions are $\mathrm{H}^{+}$and $\mathrm{O}^{+}$. The ion current collected by the probe surface per unit plasma density is found to be a strong function of ion composition. The calibration of the collected current to an absolute density is discussed, and the performance of the spherical probe is compared to other in situ instruments on board the C/NOFS satellite. The application of the calibration is discussed with respect to future fixed-bias probes; in particular, it is demonstrated that some density fluctuations will be suppressed in the collected current if the plasma composition rapidly changes along with density. This is illustrated in the observation of plasma density enhancements on C/NOFS. [http://dx.doi.org/10.1063/1.4766333]
\end{abstract}

\section{INTRODUCTION}

A commonly used tool for plasma diagnostics in the upper atmosphere is the Langmuir probe. ${ }^{1}$ The technique typically consists of sweeping the bias of a small metallic probe (typically a sphere or a cylinder) relative to the plasma potential to infer such parameters as plasma density and temperature. ${ }^{2}$ Langmuir probes have flown on numerous satellite missions for the diagnosis of space plasmas, including Atmospheric Explorer, ${ }^{3}$ Dynamics Explorer 2, ${ }^{4}$ and the Pioneer Venus Orbiter. ${ }^{5}$ Another common application is to maintain a fixed bias on the probe in either the ion or electron saturation regime in order to provide a rapid measurement of density fluctuations. ${ }^{6}$ This paper will focus on the ion saturation mode, where a negative bias is applied to ensure that only ions are collected.

The Langmuir probe on the Communication/Navigation Outage Forecast System (C/NOFS) satellite, described in Sec. II, is a fixed-bias probe that measures the total ion density in the topside equatorial ionosphere. This region typically consists of a mixture of $\mathrm{O}^{+}$and $\mathrm{H}^{+}$ions (and to a lesser extent, $\mathrm{He}^{+}$and $\mathrm{N}^{+}$). The transition height between $\mathrm{O}^{+}$and lighter ions has been studied extensively as a function of solar activity. ${ }^{7}$ The nightside transition height near the equator is found around $600 \mathrm{~km}$ during a typical solar minimum, but can be lower than $500 \mathrm{~km}$ during the extreme solar minimum of $2008 .{ }^{8}$ During a typical solar maximum $(F 10.7=200 \mathrm{sfu})$, this height can be well above $800 \mathrm{~km} .{ }^{9}$ Additionally, the dayto-day variation of ionospheric composition in the range of the C/NOFS satellite is significant. ${ }^{10}$ The effect of ion composition on the expected collected current is discussed in Sec. III.

A calibration technique for a small spherical probe in a cold collisionless plasma where composition is known is presented in Sec. IV, along with the application of this technique to the probe on C/NOFS. The performance of the calibration

a)Electronic mail: jeffrey.klenzing @ nasa.gov. is discussed in Sec. V, as well as the implication for future probe designs.

\section{INSTRUMENT DESCRIPTION}

The C/NOFS satellite was launched in April 2008 into a $13^{\circ}$ inclination orbit with perigee near $400 \mathrm{~km}$ and apogee near $860 \mathrm{~km}$. C/NOFS is equipped with multiple instrument suites designed to study the ion and neutral populations and their effect on the propagation of communication signals. ${ }^{11}$ The main focus of this paper will be the fixed-bias Langmuir probe (referred to as the Trigger Probe, or TP), which is part of the Vector Electric Field Instrument (VEFI) suite. ${ }^{12}$

The trigger probe on the VEFI is a fixed-bias partialspherical Langmuir probe designed to measure relative plasma density fluctuations, as seen in Figure 1. The probe consists of a collector and a guard element which together form a sphere with a radius of $1.25 \mathrm{~cm}$. The guard is included to shield the bulk of the collector from effects of the support boom breaking the spherical symmetry and is held at the same potential as the collector. The collector is a partial sphere with a half-angle of $111.2^{\circ}$ and has a total surface area of $13.35 \mathrm{~cm}^{2}$. Both the collector and the guard are coated in TiN. The probe is mounted so that the support boom is perpendicular to the satellite ram velocity. The boom is $0.5 \mathrm{~m}$ long. The probe can be set to one of the four bias settings: $-1.25,-2.5$, -3.75 , and $-5 \mathrm{~V}$. For these settings, the probe is well within the ion saturation region. The normal operating mode of the probe is $-3.75 \mathrm{~V}$.

The probe is primarily used as a trigger for the burst memory on board C/NOFS to save high-resolution ( $>2000$ samples/s) electric field and density data. Nominally the probe collects data at 16 samples/s. As the C/NOFS satellite has been operational for more than four years, a large statistical database of ion densities is available for study.

Several other instruments on board C/NOFS are employed in the calibration procedure. The Planar Langmuir 


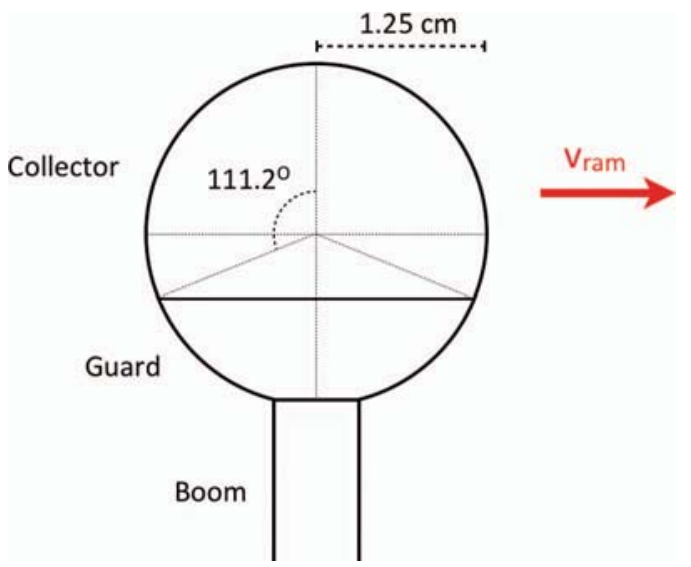

FIG. 1. The trigger probe (TP) for the VEFI suite on the C/NOFS satellite. The collector and guard form a spherical probe, with the guard removing the non-uniformities introduced by the boom.

Probe (PLP) built by the Air Force Research Laboratory (AFRL) consists of a swept Langmuir probe and an ion trap. ${ }^{13}$ The ion trap is used to provide absolute densities for calibration. Additionally, the Ion Velocity Meter (IVM), built by the University of Texas at Dallas, provides relative ion composition data using the well-known retarding potential analysis technique. ${ }^{14}$

\section{PROBE THEORY}

The equations describing the current collected by various probe geometries for different ion distributions have been discussed at length elsewhere. ${ }^{15,16}$ Here we will follow the technique of Hoegy and Brace. ${ }^{16}$ The ion current collected by a sphere immersed in a thermal (Maxwellian) plasma consisting of a single species of ion is

$$
I_{\text {random }}=q_{0} A_{\text {probe }} N_{i} \sqrt{\frac{k_{B} T_{i}}{2 \pi m_{i}}}
$$

where $q_{0}$ is the fundamental charge, $A_{\text {probe }}$ is the surface area of the sphere, $N_{i}$ is the total ion density, $k_{B}$ is the Boltzmann constant, $T_{i}$ is the ion temperature, and $m_{i}$ is the ion mass. If the sphere is biased relative to the plasma (at $\Phi_{a p p}$ ) or moving at velocity $u_{0}$ relative to the plasma, the modified current can be described by

$$
I_{\text {sphere }}=I_{\text {random }}\left[\frac{1}{2} e^{-r^{2}}+\frac{\left(\eta+r^{2}+\frac{1}{2}\right)}{r} \frac{\sqrt{\pi}}{2} \operatorname{erf}(r)\right] \text {, }
$$

where

$$
\begin{gathered}
r=\frac{u_{0}}{\sqrt{2 k_{B} T_{i} / m_{i}}}, \\
\eta=\frac{q_{0} \Phi_{a p p}}{k_{B} T_{i}} .
\end{gathered}
$$

Note that as the velocity $\left(u_{0}\right)$ approaches zero, the error function can be approximated by a Taylor expansion, such that

$$
\lim _{r \rightarrow 0} \frac{\operatorname{erf}(r)}{r}=\lim _{r \rightarrow 0} \frac{1}{r} \frac{2}{\sqrt{\pi}}\left(r-\frac{r^{3}}{3}+\frac{r^{5}}{10}-\cdots\right)=\frac{2}{\sqrt{\pi}} .
$$

Equation (2) then reduces to the usual orbital motion limited collection equation

$$
I_{\text {sphere }}=I_{\text {random }}(1+\eta) .
$$

For the C/NOFS satellite, the ion temperatures encountered during the extreme solar minimum are between $600 \mathrm{~K}$ and $1300 \mathrm{~K}^{17}$ and slowly increasing through 2011 as solar activity rises. The spacecraft velocity is $\sim 7.5 \mathrm{~km} / \mathrm{s}$, thus expected values of $r$ are $\sim 1-2$ for $\mathrm{H}^{+}$and $>6$ for $\mathrm{O}^{+}$. The probe is biased negatively so that the electron current is negligible. Further discussion of the ion collection for other cases (such as low Mach numbers and probes near the floating potential) can be found in the works of Hutchinson. ${ }^{18-20}$

For a plasma consisting of $\mathrm{H}^{+}$and $\mathrm{O}^{+}$, the average current to the sphere per unit density can be represented as

$$
\tilde{I}_{\text {sphere }}=\frac{I_{\text {sphere }}}{N_{i}}=f_{\mathrm{H}^{+}} \tilde{I}_{\mathrm{H}^{+}}+f_{\mathrm{O}^{+}} \tilde{I}_{\mathrm{O}^{+}},
$$

where $\tilde{I}_{\mathrm{H}^{+}}$and $\tilde{I}_{\mathrm{O}^{+}}$represent the average current contributions per unit density of $\mathrm{H}^{+}$and $\mathrm{O}^{+}$, and $f_{\mathrm{O}^{+}}$is the fraction of $\mathrm{O}^{+}$. Figure 2 shows these average current contributions as calculated by Eq. (2) as a function of ion temperature and reference potential for typically expected values in the range of the $\mathrm{C} / \mathrm{NOFS}$ satellite. While there is a small effect in the expected current per unit density for fluctuations in ion temperature (a)
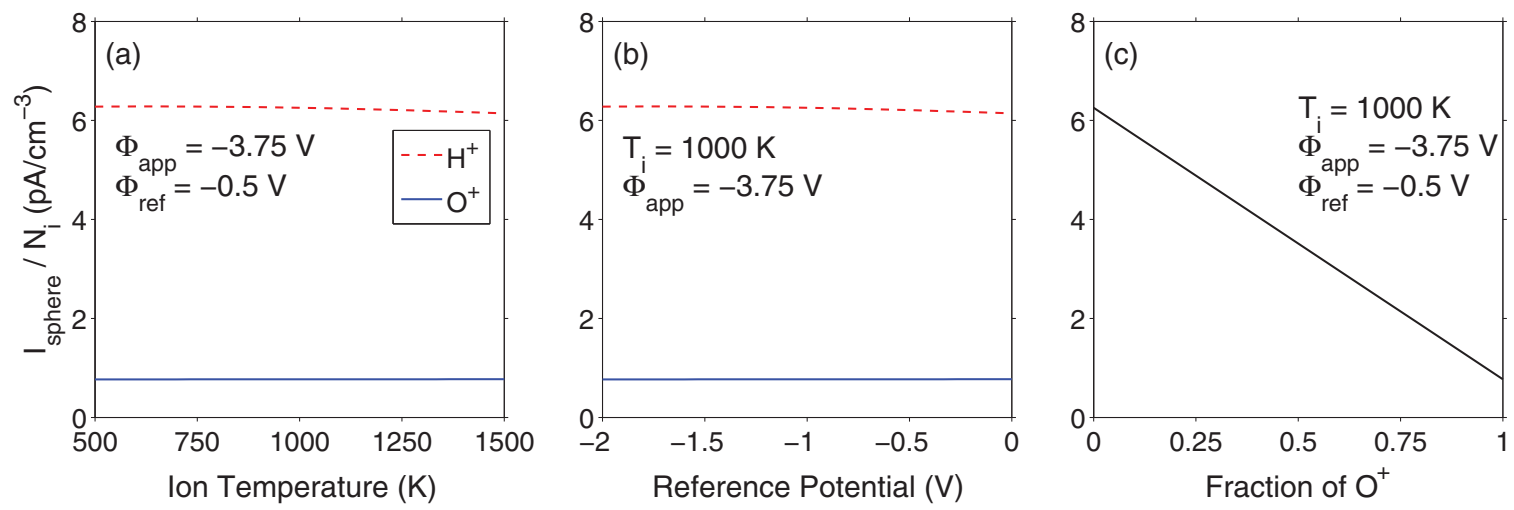

FIG. 2. The variation of expected current collected per unit density as a function of (a) ion temperature, (b) reference potential, and (c) ion composition. 
or spacecraft potential (b), these changes are commensurate with an uncertainty of a few percent in the total $\mathrm{O}^{+}$composition (c). This is because the currents due to $\mathrm{H}^{+}$and $\mathrm{O}^{+}$differ by more than a factor of six. Note that for the stationary unbiased sphere of Eq. (1), the difference in currents is expected to be only a factor of four (the square root of the ratios of masses). The additional current from $\mathrm{H}^{+}$is a result of both the negative bias of the probe and the relative speed between the probe and the plasma.

For a typical ionospheric mission, $\mathrm{O}^{+}$will be collected only on the ram surface of the probe, while light ions such as $\mathrm{H}^{+}$and $\mathrm{He}^{+}$are collected over the whole surface. This is due in part to the large thermal velocity of light ions, but it is mostly due to the effect of the negatively biased probe on the motions of the ions. Light ions that are not in the direct path of the probe are more easily accelerated toward the probe and collected on all surfaces, as demonstrated by the simulations of Sèran et al. ${ }^{21}$ Heavier ions such as $\mathrm{O}^{+}$are accelerated toward the sphere as well, but the trajectories are not changed enough for these ions to be collected on the anti-ram surface. This is illustrated in Figures 8 and 9 of Sèran et al. Additionally, it is possible under the right combination of conditions that light ions may be collected in greater abundance on the anti-ram surface of a spherical probe than on the ram surface due to this acceleration effect (see Figure 11 of the same paper).

\section{CALIBRATION TECHNIQUE}

Because most of the expected variations in $\tilde{I}_{\text {sphere }}$ come from the plasma composition for the range of temperatures encountered by C/NOFS, we can rewrite Eq. (7) as a linear function of the fraction of $\mathrm{O}^{+}$:

$$
\tilde{I}_{\text {sphere }}\left(f_{\mathrm{O}^{+}}\right)=\tilde{I}_{\mathrm{H}^{+}}+\left(\tilde{I}_{\mathrm{O}^{+}}-\tilde{I}_{\mathrm{H}^{+}}\right) f_{\mathrm{O}^{+}} .
$$

The calibration of the VEFI trigger probe utilizes a multiplicative conversion factor $\chi$, defined as

$$
N_{i}=\chi I_{\text {trigger }},
$$

where $I_{\text {trigger }}$ is the current collected in nA and $N_{i}$ is the plasma density per cubic centimeter. Note that the conversion factor $\chi$ is essentially the inverse of the effective collected current per unit density ( $\left.\tilde{I}_{\text {sphere }}\right)$. The empirical fits described below are calibrated to the total plasma density provided by the PLP ion trap and the composition information as provided by the IVM.

The value of $\chi$ is determined by taking the ratio of the TP current to the total density as provided by PLP. For three of the four bias settings, 70 consecutive days of data are chosen to give ample coverage over all altitudes and local times (the precession of the $\mathrm{C} / \mathrm{NOFS}$ perigee with respect to local time is about 65 days). The lowest bias setting $(-5 \mathrm{~V})$ only uses 26 consecutive days in 2008 and 29 consecutive days in 2010 due to the minimal time spent at this setting. The data are decimated so that one point is used every $64 \mathrm{~s}$. The number of points for each fit is reported in Table I.
TABLE I. Data used for each fit.

\begin{tabular}{lcc}
\hline \hline LPBias (V) & Days & Number of points \\
\hline-1.25 & $105-174,2009$ & 79295 \\
-2.50 & $230-299,2009$ & 138810 \\
-3.75 & 350,$2009 ; 54,2010$ & 140006 \\
-5.00 & $155-179,2008 ; 55-84,2010$ & 83340 \\
\hline \hline
\end{tabular}

The calibration factor $\chi$ is then fit to the function in Eq. (10) using a Levenburg-Marquardt ${ }^{22}$ least-squares fit,

$$
\chi\left(f_{\mathrm{O}^{+}}\right)=\frac{1}{a+b f_{\mathrm{O}^{+}}} .
$$

The results of the fitting procedure for each bias setting (referred to as LPBias) can be seen in Figure 3, where all data are plotted as a function of $f_{\mathrm{O}^{+}}$. The mean and standard deviations are plotted as error bars over the data points, and the fits are plotted as lines. The fit parameters $a$ and $b$ for all four bias settings are listed in Table II along with the corresponding coefficient of determination $\left(\mathrm{R}^{2}\right)$. All fits are performed using the MATLAB R2012a curve fitting toolbox.

Figure 4 shows the mean and standard deviations for $\chi$ from Figure 3 replotted as a function of applied potential (LPBias) for selected compositions. The changes in the measured value of $\chi$ for each bias setting are as expected. As the

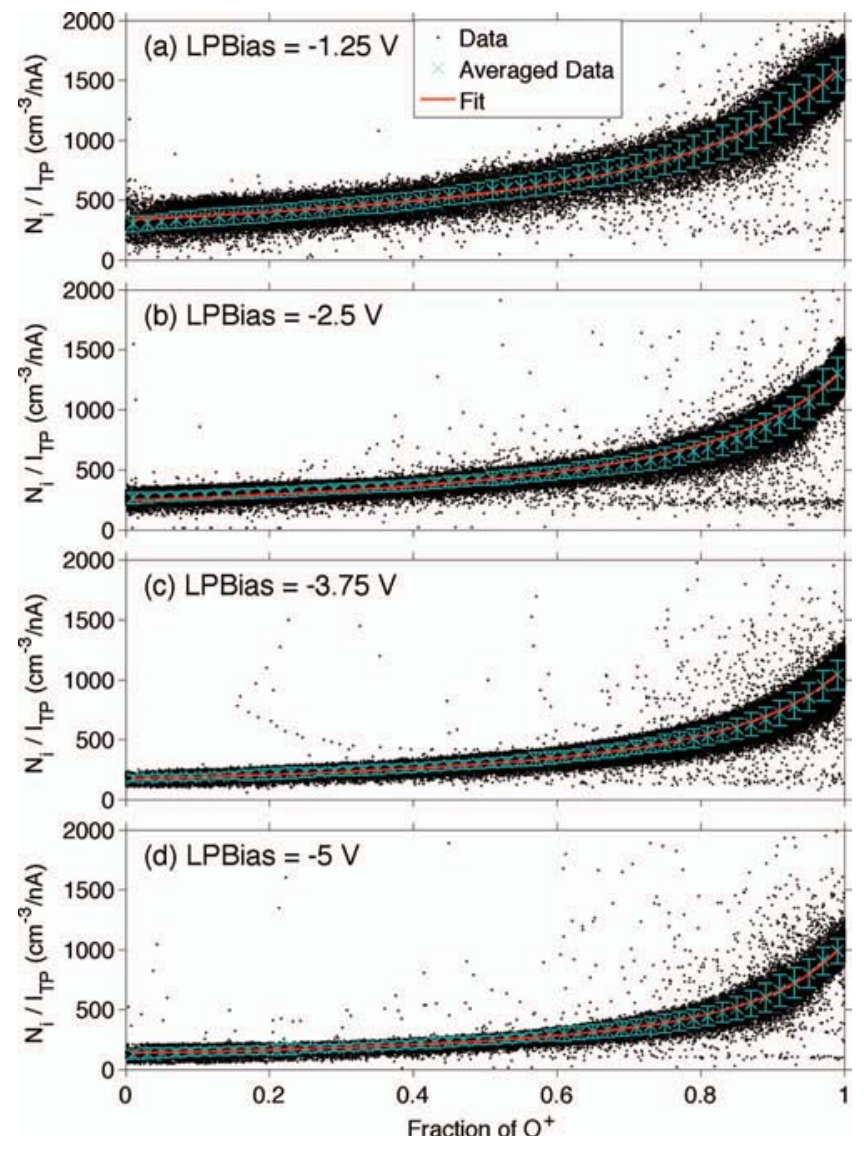

FIG. 3. The ratio of PLP density $\left(N_{i}\right)$ to trigger probe current $\left(I_{\mathrm{TP}}\right)$ as a function of the fraction of $\mathrm{O}^{+}$as measured by IVM for the four bias settings. The cyan lines represent the mean and standard deviation of the data; the red lines are the fit to the data. 
TABLE II. Fitted parameters.

\begin{tabular}{lcll}
\hline \hline LPBias (V) & $\mathrm{a}$ & \multicolumn{1}{c}{$\mathrm{b}$} & \multicolumn{1}{c}{$\mathrm{R}^{2}$} \\
\hline-1.25 & 0.002978 & -0.002371 & 0.9478 \\
-2.50 & 0.004055 & -0.00331 & 0.964 \\
-3.75 & 0.005774 & -0.004866 & 0.9638 \\
-5.00 & 0.007402 & -0.006461 & 0.9748 \\
\hline \hline
\end{tabular}

applied bias becomes more negative, more ions are attracted to the probe and the resultant current per unit density increases. This leads to an overall reduction in $\chi$. The spread in $\chi$ also decreases as the applied bias becomes large relative to any fluctuations in spacecraft potential.

\section{PERFORMANCE IN EARTH'S IONOSPHERE}

The performance of the calibrated fits is compared for all data in 2011 in Figure 5. In addition to the calibration method described above, two other "densities" are calculated using an assumption that the probe is always in an environment consisting of a single ion species $\left(\mathrm{H}^{+}\right.$or $\left.\mathrm{O}^{+}\right)$. The vertical dashed lines represent a discrepancy of 30\% between VEFI and PLP. The calibration utilizing the exact composition information typically provides densities that agree within $30 \%$ of the PLP density measurement, while the other two estimates of density may be off by as much as a factor of ten.

The implication of this work is not just that ion composition is vital to calculating an absolute density from a fixed-bias spherical probe, but also for calculating relative density fluctuations for satellites near the $\mathrm{H}^{+} / \mathrm{O}^{+}$transition height. To illustrate this, Figure 6 shows a single orbit where the C/NOFS satellite passes through a series of plasma density enhancements. These structures are frequently encountered by $\mathrm{C} / \mathrm{NOFS}$ in the nighttime ionosphere and consist of a sharp increase in both the total density and the relative fraction of $\mathrm{O}^{+}{ }^{23}$ Panel (a) shows the current measured by the trigger probe during a typical encounter with these structures

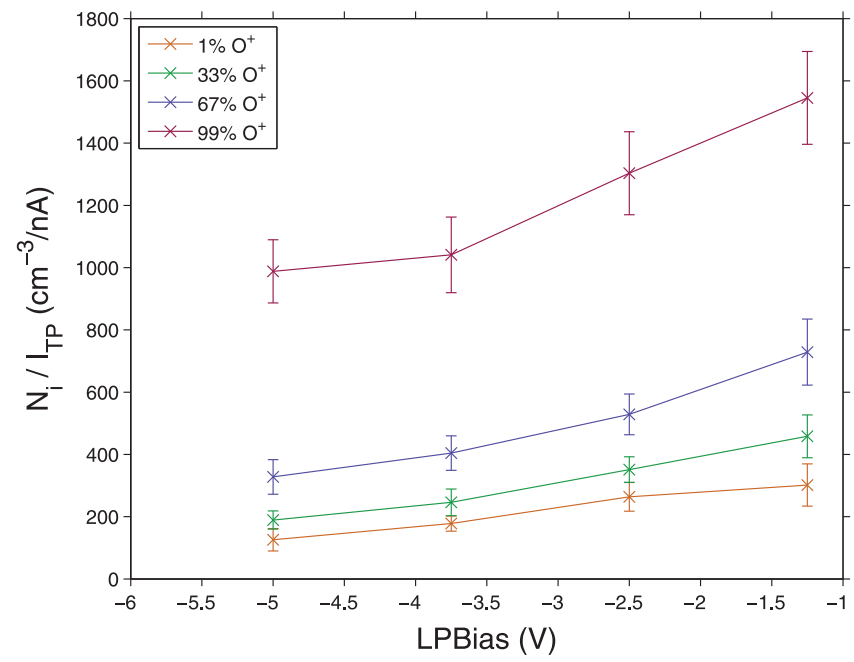

FIG. 4. The ratio of PLP density $\left(N_{i}\right)$ to trigger probe current $\left(I_{\mathrm{TP}}\right)$ as a function of the fraction of applied potential for four different levels of $\mathrm{O}^{+}$ composition.

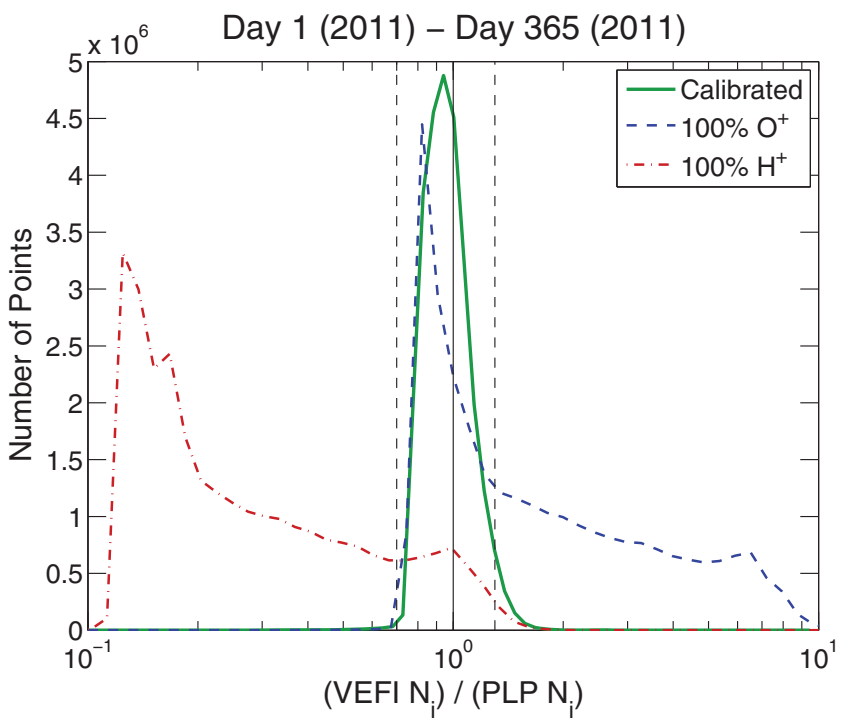

FIG. 5. Performance of the calibration method for total density for all days in 2011. The vertical dashed lines represent discrepancies of 30\% versus the PLP density.

on the night of September 15, 2009; panel (c) shows the relative composition of $\mathrm{O}^{+}$. In panel (b), the absolute densities reported by both PLP and the calibrated TP are reported as solid lines, with a third option using a constant assumption of $70 \% \mathrm{O}^{+}$to convert the TP currents to densities (referred to as the uncalibrated VEFI densities). The uncalibrated curve suppresses the density increase, leading to a loss of information. This is due to the increase in $\mathrm{O}^{+}$inside the plasma enhancement, which is collected by a spherical probe less efficiently that the $\mathrm{H}^{+}$outside the enhancement. In order to capture these density fluctuations, knowledge of the plasma composition is needed in addition to the collected current.

This suppression effect is the result of flying an ion saturation probe through a two-species plasma where the two
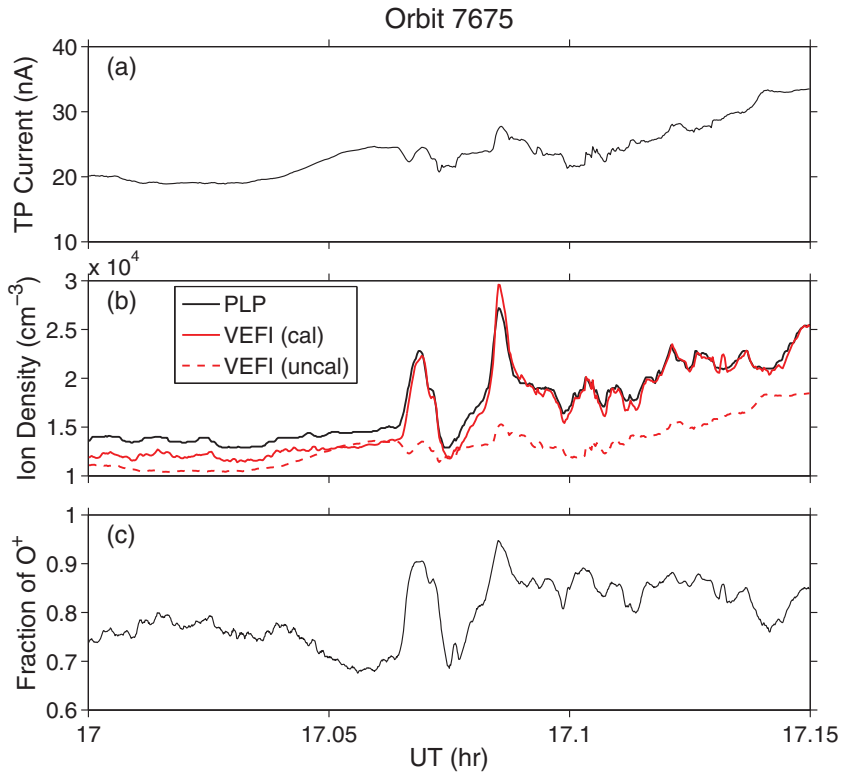

FIG. 6. An example of plasma density structures where a changing composition results in a suppression of the features if the probe is uncalibrated. 
ion species have very different Mach numbers $(r)$. For a highvelocity satellite (in this case, $u_{0} \simeq 7.5 \mathrm{~km} / \mathrm{s}$ ), this results in Mach numbers of roughly 1 and 6 for $\mathrm{H}^{+}$and $\mathrm{O}^{+}$, respectively. Because of the large spacecraft velocity, variations due to ion temperature are expected to be minimal (see Figure 2(a)). For a lower-velocity platform such as a sounding rocket (where spacecraft velocity is typically less than $1 \mathrm{~km} / \mathrm{s}$ ), this is not the case. For sounding rockets, the flow of $\mathrm{H}^{+}$and $\mathrm{O}^{+}$will typically be subsonic $(r<1)$, and now fluctuations in ion temperature are expected to have a significant effect on the effective current collected. A suppression of density fluctuations similar to that observed on C/NOFS may occur for heavier species such as $\mathrm{Fe}^{+}$, where $r$ may be 2-3.

\section{SUMMARY}

The calibration technique for the spherical fixed-bias Langmuir probe for the VEFI on C/NOFS is described. It is found that the highly-variable ion composition in the vicinity of the satellite strongly affects the current collected by a spherical probe for a given density. The calibration of the probe is greatly improved by incorporating in situ measurements of ion composition as provided by the IVM instrument. Additionally, there are events such as plasma density enhancements where the changes in total density and relative ion composition combine in such a way that the probe current is relatively unchanged while the ion density doubles in magnitude. This is an important consideration for similar ion saturation probes designed to look at relative fluctuations near the $\mathrm{H}^{+} / \mathrm{O}^{+}$transition height. For probes well above this transition height (and therefore in a single species plasma), relative density fluctuations can be reliably determined by a fixedbias probe. However, knowledge of the in situ composition is essential in truly calibrating a probe in a multi-component plasma.

\section{ACKNOWLEDGMENTS}

The Communication/Navigation Outage Forecast System (C/NOFS) mission, conceived and developed by the Air Force Research Laboratory (AFRL), is sponsored and executed by the USAF Space Test Program. Thanks to F. Simões for use- ful comments on this manuscript and P. A. Roddy of AFRL and R. A. Heelis of UT Dallas for providing data for the calibration procedure.

${ }^{1}$ L. H. Brace, in Measurement Techniques in Space Plasmas: Particles, edited by R. Pfaff, J. E. Borovsky, and D. T. Young (AGU, Washington, DC, 1998), pp. 23-35.

${ }^{2}$ H. Mott-Smith and I. Langmuir, Phys. Rev. 28, 727-763 (1926).

${ }^{3}$ L. H. Brace, R. F. Theis, and A. Dalgarno, Radio Sci. 8, 341-348, doi:10.1029/RS008i004p00341 (1973).

${ }^{4}$ J. P. Krehbiel, L. H. Brace, R. F. Theis, W. H. Pinkus, and R. B. Kaplan, Space Sci. Instrum. 5, 493-502 (1981).

${ }^{5}$ L. H. Brace, W. R. Hoegy, and R. F. Theis, J. Geophys. Res. 93, 7282-7296, doi:10.1029/JA093iA07p07282 (1988).

${ }^{6}$ W. R. Hoegy, S. A. Curtis, L. H. Brace, N. C. Maynard, and R. A. Heelis, Geophys. Res. Lett. 9(9), 993-996, doi:10.1029/GL009i009p00993 (1982).

${ }^{7}$ L. Triskova, V. Truhlik, and J. Smilauer, Adv. Space Res. 31, 653-663 (2003).

${ }^{8}$ R. A. Heelis, W. R. Coley, A. G. Burrell, M. R. Hairston, G. D. Earle, M. D. Perdue, R. A. Power, L. L. Harmon, B. J. Holt, and C. R. Lippincott, Geophys. Res. Lett. 36, L00C03, doi:10.1029/2009GL038652 (2009).

${ }^{9}$ K. H. West, R. A. Heelis, and F. J. Rich, J. Geophys. Res. 102, 295-305, doi:10.1029/96JA03031 (1997).

${ }^{10}$ J. Klenzing, F. Simões, S. Ivanov, R. A. Heelis, D. Bilitza, R. Pfaff, and D. Rowland, J. Geophys. Res. 116, A12330, doi:10.1029/2011JA017213 (2011).

${ }^{11}$ O. de La Beaujardière, J. Atmos. Solar-Ter. Phys. 66, 1573-1591 (2004).

${ }^{12}$ R. Pfaff, D. Rowland, H. Freudenreich, K. Bromund, G. Le, M. H. Acuña, J. Klenzing, M. C. Liebrecht, S. Martin, W. J. Burke, N. C. Maynard, D. E. Hunton, P. A. Roddy, J. O. Ballenthin, and G. R. Wilson, J. Geophys. Res. 115, A12324, doi:10.1029/2010JA016023 (2010).

${ }^{13}$ P. A. Roddy, D. E. Hunton, J. O. Ballenthin, and K. M. Groves, J. Geophys. Res. 115, A06303, doi:10.1029/2010JA015288 (2010).

${ }^{14}$ R. A. Heelis and W. B. Hanson, in Measurement Techniques in Space Plasmas: Particles, edited by R. Pfaff, J. E. Borovsky, and D. T. Young (AGU, Washington, DC, 1998), pp. 61-71.

${ }^{15}$ J. G. Laframboise and L. J. Sonmor, J. Geophys. Res. 98, 337-357, doi:10.1029/92JA00839 (1993).

${ }^{16}$ W. Hoegy and L. H. Brace, Rev. Sci. Instrum. 70, 3015-3024 (1999).

${ }^{17}$ W. R. Coley, R. A. Heelis, M. R. Hairston, G. D. Earle, M. D. Perdue, R. A. Power, L. L. Harmon, B. J. Holt, and C. R. Lippincott, J. Geophys. Res. 115, A02313, doi:10.1029/2009JA014665 (2010).

${ }^{18}$ I. H. Hutchinson, Plasma Phys. Controlled Fusion 44, 1953-1977 (2002).

${ }^{19}$ I. H. Hutchinson, Plasma Phys. Controlled Fusion 45, 1477-1500 (2003).

${ }^{20}$ I. H. Hutchinson, Plasma Phys. Controlled Fusion 47, 71-87 (2005).

${ }^{21}$ E. Séran, J. J. Berthelier, F. Saouri, and J. P. Lebreton, Ann. Geophys. 23, 1723-1733 (2005).

${ }^{22}$ D. W. Marquardt, J. Soc. Ind. Appl. Math. 2, 431-441 (1963).

${ }^{23}$ J. H. Klenzing, D. E. Rowland, R. F. Pfaff, G. Le, H. Freudenreich, R. A. Haaser, A. G. Burrell, R. A. Stoneback, W. R. Coley, and R. A. Heelis, J. Geophys. Res. 116, A09324, doi:10.1029/2011JA016711 (2011). 